\title{
Test for Discontinuities in Nonparametric Regression ${ }^{\dagger}$
}

\author{
Dongryeon Park ${ }^{1)}$
}

\begin{abstract}
The difference of two one-sided kernel estimators is usually used to detect the location of the discontinuity points of regression function. The large absolute value of the statistic imply discontinuity of regression function, so we may use the difference of two one-sided kernel estimators as the test statistic for testing null hypothesis of a smooth regression function. The problem is, however, we only know the asymptotic distribution of the test statistic under $H_{0}$ and we hardly expect the good performance of test if we rely solely on the asymptotic distribution for determining the critical points. In this paper, we show that if we adjust the bias of test statistic properly, the asymptotic rules hold for even small sample size situation.
\end{abstract}

Keywords: Asymptotic distribution; discontinuity points; nonparametric regression.

\section{Introduction}

The problem of detecting discontinuity points in nonparametric regression has received much attention over the years. The literature on this topic is quite large. Refer to Qiu (2005) for the detail. Much of these research has focused on the estimation, either of the location of the discontinuity points or of the regression function with a finite number of jump discontinuities.

When we know the regression function has discontinuity points, we would estimate first the location of the discontinuity points and then apply the smoothing technique to each smooth parts of regression function separately. This leads to a discontinuous estimate, which is usually quite different from the continuous estimate for the case of assuming the continuous regression function. Therefore when we estimate regression function, we need to know whether it is reasonable to assume that the regression function is continuous. This is about the testing problem for the presence of discontinuities.

Relatively less attention has been paid for testing the null hypothesis of a smooth regression function. Wu and Chu (1993) proposed a test based on asymptotic distribution of the estimator of jump sizes, and demonstrated the performance of their test by simulation study, but the performance of the test at the sample size $n=50$ was not good at all. The empirical Type I error was too high. Müller and Stadtmüller (1999) considered a test statistic based on sum of squared differences of the data and developed asymptotic distribution of test statistic to allow a test for the presence of jumps. The finite sample behavior of their test procedure is pretty excellent for $n=1000$ case, but

$\dagger$ This work has been supported by Hanshin University Research Grant.

1) Professor, Department of Statistics, Hanshin University, 441 Yangsan-Dong, Osan, Kyunggi-Do 447-791, Korea. E-mail: drpark@hs.ac.kr. 
the test has relatively low power and large empirical Type I error at both $n=30$ and $n=100$.

The test statistic of Gijbels and Goderniaux (2004a) is the difference of two one-sided kernel estimators, which is usually used for detecting the location of jump points. They proposed to use a bootstrap procedure to approximate the critical points of the test. They developed the bootstrap procedure just because they do not want to depend on asymptotic law for determining the critical points of the test. It is well known that we may have a discrepancy between the large sample result and the finite sample result, especially at the small sample size. Thus it is not recommendable to rely solely on the asymptotic distribution of the test statistic for computing the critical points of the test at the small sample size situation.

In this paper, we discuss the testing problem for the null hypothesis of a smooth regression function. The test statistic we consider is just identical with that of Gijbels and Goderniaux (2004a). The asymptotic distribution of the test statistic, however, is used for determining the critical points of the test. We show that with proper adjustment the asymptotic result hold for even small sample size situation.

The paper is organized as follows. In Section 2, we propose test statistics which are based on the statistic of detecting the location of jump points. The main difference among these test statistics is the bias adjustment term. The simulation results for evaluating the proposed testing procedures are presented in Section 3. In Section 4, we provide some concluding remarks and further research topics.

\section{Testing Procedures}

We consider the regression model

$$
Y_{i}=m\left(x_{i}\right)+\epsilon_{i}, \quad i=1, \ldots, n,
$$

where $m$ is a unknown regression function which is assumed to be smooth, except possibly at a finite number of discontinuity points. Throughout the paper we assume that $m$ is defined on the interval $[0,1]$ without loss of generality and the design points $x_{i}$ are equally spaced on $[0,1]$. The error $\epsilon_{i}$ are assumed to be independent with mean 0 and finite variance $\sigma^{2}$.

We want to test whether the unknown regression function $m$ has at least one jump discontinuity. More formally, we consider the testing problem

$$
\begin{array}{ll}
H_{0}: m_{+}(x)=m_{-}(x) & \forall x \in[0,1], \\
H_{1}: m_{+}(x) \neq m_{-}(x) & \exists x \in[0,1],
\end{array}
$$

where $m_{+}(x)$ and $m_{-}(x)$ denote the right and left limits of $m$ at the point $x$. Under the null hypothesis, we also assume that $m_{+}^{\prime}(x)=m_{-}^{\prime}(x)$ and $m_{+}^{\prime \prime}(x)=m_{-}^{\prime \prime}(x)$ for all $x \in(0,1)$.

We consider the following statistic which is the difference of two one-sided kernel estimators:

$$
T=\hat{m}_{+}\left(\hat{x}_{0}\right)-\hat{m}_{-}\left(\hat{x}_{0}\right),
$$


where

$$
\hat{m}_{+}\left(\hat{x}_{0}\right)=\sum_{i=1}^{n} Y_{i} K_{1}\left(\frac{x_{i}-\hat{x}_{0}}{h}\right) / \sum_{i=1}^{n} K_{1}\left(\frac{x_{i}-\hat{x}_{0}}{h}\right)
$$

and

$$
\hat{m}_{-}\left(\hat{x}_{0}\right)=\sum_{i=1}^{n} Y_{i} K_{2}\left(\frac{x_{i}-\hat{x}_{0}}{h}\right) / \sum_{i=1}^{n} K_{2}\left(\frac{x_{i}-\hat{x}_{0}}{h}\right)
$$

and $h$ is a smoothing parameter and $\hat{x}_{0}$ is defined by the maximizer of $|T|$ over $x \in$ $[h, 1-h]$. Namely,

$$
\hat{x}_{0}=\arg \max _{x \in[h, 1-h]}\left|\hat{m}_{+}(x)-\hat{m}_{-}(x)\right| .
$$

$K_{1}$ and $K_{2}$ are two kernel functions satisfying the following conditions:

1. The support of $K_{1}$ is $[0,1]$ and the support of $K_{2}$ is $[-1,0)$.

2. $K_{j}(x) \geq 0, \quad j=1,2$.

3. $\int_{-1}^{1} K_{j}(x) d x=1, \quad j=1,2$.

Originally, the statistic $T$ was proposed to detect the discontinuity points of the regression function. See Qiu (2005) for detail. When we assume there is only one discontinuity point, the location of the discontinuity point is estimated by $\hat{x}_{0}$ and the jump magnitude at the point is estimated by $|T|$. Therefore the large value of $|T|$ can be used as the evidence against $H_{0}$. This means that we can reject $H_{0}$ if we obtain too large value of $|T|$. Thus we can use $T$ as a test statistic.

In order to determine the critical points for $T$, we should know the distribution of $T$ under the null hypothesis. The asymptotic distribution of $T$ under the null hypothesis is derived in Qiu (2005). Under some regularity conditions, $T$ is asymptotically normal with the mean

$$
E(T)=o\left(h^{2}\right)
$$

and the variance

$$
\operatorname{Var}(T)=\frac{2 \sigma^{2} K_{2}^{(1)}}{\left(K_{1}^{(1)}\right)^{2} n h}+o\left(\frac{1}{n h}\right)
$$

where

$$
K_{j}^{(1)}=\int_{0}^{1} K_{1}^{j}(u) d u, \quad j=1,2 .
$$

Therefore if we want to rely solely on asymptotic law for determining the critical points, then we can construct the testing procedure as follows: Reject $H_{0}$ if

$$
\left|T_{1}\right| \geq z_{1-\frac{\alpha}{2}}
$$

where

$$
T_{1}=T / \frac{2 \hat{\sigma}^{2} K_{2}^{(1)}}{\left(K_{1}^{(1)}\right)^{2} n h}
$$


and $z_{1-\alpha / 2}$ is $100(1-\alpha / 2)^{t h}$ percentile of the standard normal distribution. Several estimators of $\sigma^{2}$ are available. The trimmed mean version of $\hat{\sigma}^{2}$ used in $\mathrm{Wu}$ and Chu (1993) is defined as follows:

$$
\hat{\sigma}^{2}=\frac{1}{2(n-1-2 q)} \sum_{i=2+q}^{n-q} \xi_{i},
$$

where $\xi_{i}$ denote the rearranged $\left(Y_{i}-Y_{i-1}\right)^{2}$ in ascending order. The number $q$ is determined by the experimenter.

The asymptotic distribution of $T$ under the null hypothesis can be refined by the exact computation of the mean and the variance. The mean of $T$ is given by

$$
\begin{aligned}
E(T)= & E\left(\hat{m}_{+}\left(\hat{x}_{0}\right)-\hat{m}_{-}\left(\hat{x}_{0}\right)\right) \\
= & \frac{\sum_{i=1}^{n} E\left(Y_{i}\right) K_{1 h}\left(x_{i}-\hat{x}_{0}\right)}{\sum_{i=1}^{n} K_{1 h}\left(x_{i}-\hat{x}_{0}\right)}-\frac{\sum_{i=1}^{n} E\left(Y_{i}\right) K_{2 h}\left(x_{i}-\hat{x}_{0}\right)}{\sum_{i=1}^{n} K_{2 h}\left(x_{i}-\hat{x}_{0}\right)},
\end{aligned}
$$

where

$$
K_{j h}\left(x_{i}-\hat{x}_{0}\right)=K_{j}\left(\frac{x_{i}-\hat{x}_{0}}{h}\right), \quad j=1,2
$$

Under $H_{0}$, we can get the following expression by using the first order approximation of Taylor series expansion for $m(x)$.

$$
E(T)=c_{1} \cdot m^{\prime}\left(\hat{x}_{0}\right),
$$

where

$$
c_{1}=\frac{\sum_{i=1}^{n}\left(x_{i}-\hat{x}_{0}\right) K_{1 h}\left(x_{i}-\hat{x}_{0}\right)}{\sum_{i=1}^{n} K_{1 h}\left(x_{i}-\hat{x}_{0}\right)}-\frac{\sum_{i=1}^{n}\left(x_{i}-\hat{x}_{0}\right) K_{2 h}\left(x_{i}-\hat{x}_{0}\right)}{\sum_{i=1}^{n} K_{2 h}\left(x_{i}-\hat{x}_{0}\right)} .
$$

Under $H_{0}$, we also can get the following result by using the second order approximation of Taylor series expansion for $m(x)$.

$$
E(T)=c_{1} \cdot m^{\prime}\left(\hat{x}_{0}\right)+c_{2} \cdot m^{\prime \prime}\left(\hat{x}_{0}\right),
$$

where

$$
c_{2}=\frac{\sum_{i=1}^{n}\left(x_{i}-\hat{x}_{0}\right)^{2} K_{1 h}\left(x_{i}-\hat{x}_{0}\right)}{2 \sum_{i=1}^{n} K_{1 h}\left(x_{i}-\hat{x}_{0}\right)}-\frac{\sum_{i=1}^{n}\left(x_{i}-\hat{x}_{0}\right)^{2} K_{2 h}\left(x_{i}-\hat{x}_{0}\right)}{2 \sum_{i=1}^{n} K_{2 h}\left(x_{i}-\hat{x}_{0}\right)} .
$$

It is clear that the variance of $T$ is given by

$$
\operatorname{Var}(T)=c_{3} \cdot \sigma^{2},
$$


where

$$
c_{3}=\frac{\sum_{i=1}^{n} K_{1 h}^{2}\left(x_{i}-\hat{x}_{0}\right)}{\left\{\sum_{i=1}^{n} K_{1 h}\left(x_{i}-\hat{x}_{0}\right)\right\}^{2}}+\frac{\sum_{i=1}^{n} K_{2 h}^{2}\left(x_{i}-\hat{x}_{0}\right)}{\left\{\sum_{i=1}^{n} K_{2 h}\left(x_{i}-\hat{x}_{0}\right)\right\}^{2}} .
$$

By using the expression (2.3), (2.4) and (2.5), we can construct test statistic $T_{2}$ and $T_{3}$ as follows:

$$
\begin{aligned}
T_{2} & =\frac{T-c_{1} \cdot \hat{m}^{\prime}\left(\hat{x}_{0}\right)}{\sqrt{c_{3} \cdot \hat{\sigma}^{2}}}, \\
T_{3} & =\frac{T-c_{1} \cdot \hat{m}^{\prime}\left(\hat{x}_{0}\right)-c_{2} \cdot \hat{m}^{\prime \prime}\left(\hat{x}_{0}\right)}{\sqrt{c_{3} \cdot \hat{\sigma}^{2}}} .
\end{aligned}
$$

Under $H_{0}$, the distribution of both $T_{2}$ and $T_{3}$ is a standard normal distribution. As we check out during the simulation study, it turns out that the asymptotic $\operatorname{Var}(T)$ in (2.2) and $\operatorname{Var}(T)$ by the exact computation in (2.5) produce almost the same value for all cases. Therefore, the key difference among $T_{1}, T_{2}$ and $T_{3}$ is the bias adjustment. $T_{1}$ has no bias adjustment, $T_{2}$ has a bias adjustment term based on the first order approximation and $T_{3}$ has bias adjustment terms based on the first and second order approximation. These minor adjustment can produce a surprising change.

\section{A Simulation Study}

A simulation study was conducted to evaluate the finite sample performance of the proposed testing procedure. We consider the following regression functions:

$$
\begin{aligned}
& m_{1}(x)=2 x+d \cdot I(x>0.5), \\
& m_{2}(x)=7 x+d \cdot I(x>0.5), \\
& m_{3}(x)=2 x^{2}+d \cdot I(x>0.5), \\
& m_{4}(x)=7 x^{2}+d \cdot I(x>0.5),
\end{aligned}
$$

where $d$ is a jump magnitude and we consider $d=0,1,2,3$ for each model. The design points were given by $x_{i}=(i-1) /(n-1)$ for $i=1, \ldots, n$. The error terms were generated from $N(0,0.5)$. The sample size considered were $n=50,100$. The first and second order derivative estimator of $m(x)$ for $T_{2}$ and $T_{3}$ were evaluated by the function glkerns of the package lokern in R.

The smoothing parameter plays very important role in evaluating $\hat{x}_{0}, T_{2}$ and $T_{3}$. For $\hat{x}_{0}$, we may use the bootstrap procedure for selecting bandwidth proposed in Gijbels and Goderniaux (2004b) and for $\hat{m}^{\prime}\left(\hat{x}_{0}\right)$ and $\hat{m}^{\prime \prime}\left(\hat{x}_{0}\right)$, we also may use the cross-validation method. However, we do not want to add any uncertainty to the simulation results because of any possible wrong choice of smoothing parameters. Besides, if we use several different smoothing parameters, we can see the effect of the smoothing parameter to performance of each test statistic. Thus we used 21 different smoothing parameter from $h=0.05$ to $h=0.25$ by 0.01 for each data set. 
Table 3.1: Simulation results for $m_{1}$ and $m_{2}$.

\begin{tabular}{|c|c|c|c|c|c|c|c|c|}
\hline & \multirow{2}{*}{$d$} & \multirow{2}{*}{$h$} & \multicolumn{3}{|c|}{$n=50$} & \multicolumn{3}{|c|}{$n=100$} \\
\hline & & & $T_{1}$ & $T_{2}$ & $T_{3}$ & $T_{1}$ & $T_{2}$ & $T_{3}$ \\
\hline \multirow{20}{*}{$m_{1}$} & \multirow{5}{*}{0} & .05 & .936 & .033 & .001 & .992 & .123 & .086 \\
\hline & & .10 & .872 & .049 & .035 & .961 & .092 & .082 \\
\hline & & .15 & .898 & .042 & .035 & .984 & .072 & .070 \\
\hline & & .20 & .918 & .034 & .031 & .992 & .055 & .055 \\
\hline & & .25 & .932 & .032 & .029 & 1.00 & .049 & .048 \\
\hline & \multirow{5}{*}{1} & .05 & .960 & .043 & .001 & .997 & .142 & .092 \\
\hline & & .10 & .977 & .067 & .044 & .999 & .151 & .141 \\
\hline & & .15 & .998 & .061 & .056 & 1.00 & .174 & .163 \\
\hline & & .20 & 1.00 & .070 & .062 & 1.00 & .245 & .238 \\
\hline & & .25 & 1.00 & .103 & .096 & 1.00 & .310 & .307 \\
\hline & \multirow{5}{*}{2} & .05 & .991 & .066 & .001 & 1.00 & .239 & .180 \\
\hline & & .10 & 1.00 & .156 & .112 & 1.00 & .494 & .463 \\
\hline & & .15 & 1.00 & .249 & .219 & 1.00 & .720 & .707 \\
\hline & & .20 & 1.00 & .397 & .371 & 1.00 & .868 & .865 \\
\hline & & .25 & 1.00 & .537 & .523 & 1.00 & .942 & .938 \\
\hline & \multirow{5}{*}{3} & .05 & .999 & .184 & .023 & 1.00 & .556 & .455 \\
\hline & & .10 & 1.00 & .488 & .400 & 1.00 & .897 & .883 \\
\hline & & .15 & 1.00 & .715 & .673 & 1.00 & .984 & .981 \\
\hline & & .20 & 1.00 & .866 & .859 & 1.00 & .998 & .999 \\
\hline & & .25 & 1.00 & .951 & .947 & 1.00 & 1.00 & 1.00 \\
\hline \multirow{20}{*}{$m_{2}$} & \multirow{5}{*}{0} & .05 & .979 & .045 & .001 & .999 & .121 & .076 \\
\hline & & .10 & .998 & .051 & .036 & 1.00 & .103 & .085 \\
\hline & & .15 & 1.00 & .037 & .028 & 1.00 & .085 & .073 \\
\hline & & .20 & 1.00 & .033 & .031 & 1.00 & .063 & .061 \\
\hline & & .25 & 1.00 & .029 & .024 & 1.00 & .054 & .054 \\
\hline & \multirow{5}{*}{1} & .05 & .992 & .036 & .002 & 1.00 & .126 & .085 \\
\hline & & .10 & 1.00 & .059 & .030 & 1.00 & .133 & .126 \\
\hline & & .15 & 1.00 & .076 & .063 & 1.00 & .175 & .170 \\
\hline & & .20 & 1.00 & .091 & .086 & 1.00 & .214 & .212 \\
\hline & & .25 & 1.00 & .091 & .092 & 1.00 & .262 & .262 \\
\hline & \multirow{5}{*}{2} & .05 & 1.00 & .072 & .007 & 1.00 & .241 & .182 \\
\hline & & .10 & 1.00 & .164 & .119 & 1.00 & .477 & .445 \\
\hline & & .15 & 1.00 & .283 & .253 & 1.00 & .714 & .702 \\
\hline & & .20 & 1.00 & .410 & .387 & 1.00 & .876 & .872 \\
\hline & & .25 & 1.00 & .529 & .514 & 1.00 & .938 & .938 \\
\hline & \multirow{5}{*}{3} & .05 & 1.00 & .172 & .025 & 1.00 & .538 & .448 \\
\hline & & .10 & 1.00 & .458 & .357 & 1.00 & .893 & .862 \\
\hline & & .15 & 1.00 & .660 & .610 & 1.00 & .993 & .994 \\
\hline & & .20 & 1.00 & .834 & .809 & 1.00 & 1.00 & 1.00 \\
\hline & & .25 & 1.00 & .934 & .931 & 1.00 & 1.00 & 1.00 \\
\hline
\end{tabular}

The simulation results are presented in Table 3.2 and 3.2. In each table, we report the proportion of times that the null hypothesis is rejected in 1000 simulation samples. The significance level is $\alpha=0.05$. We report only the case of $h=0.05,0.1,0.15,0.2,0.25$ to save the space.

Note that the test statistic $T_{1}$ shows the extremely poor performance. Its rejection ratio is at least $87 \%$ when $H_{0}$ is true (the case of $d=0$ ). This is why nobody use $T_{1}$ as the test statistic yet and Gijbels and Goderniaux (2004a) tried to find out the distribution of $T$ under $H_{0}$ using the bootstrap procedure. $T_{1}$ simply produces too large value.

On the other hand, note that both $T_{2}$ and $T_{3}$ show relatively good performance. We 
Table 3.2: Simulation results for $m_{3}$ and $m_{4}$.

\begin{tabular}{|c|c|c|c|c|c|c|c|c|}
\hline & \multirow{2}{*}{$d$} & \multirow{2}{*}{$h$} & \multicolumn{3}{|c|}{$n=50$} & \multicolumn{3}{|c|}{$n=100$} \\
\hline & & & $T_{1}$ & $T_{2}$ & $T_{3}$ & $T_{1}$ & $T_{2}$ & $T_{3}$ \\
\hline \multirow{20}{*}{$m_{3}$} & \multirow{5}{*}{0} & .05 & .940 & .040 & .002 & .989 & .116 & .085 \\
\hline & & .10 & .876 & .058 & .034 & .981 & .085 & .075 \\
\hline & & .15 & .907 & .055 & .045 & .993 & .082 & .074 \\
\hline & & .20 & .946 & .040 & .037 & .998 & .069 & .069 \\
\hline & & .25 & .946 & .032 & .029 & .999 & .059 & .060 \\
\hline & \multirow{5}{*}{1} & .05 & .970 & .045 & .004 & .994 & .131 & .086 \\
\hline & & .10 & .967 & .068 & .049 & 1.00 & .151 & .130 \\
\hline & & .15 & .995 & .082 & .071 & 1.00 & .173 & .176 \\
\hline & & .20 & 1.00 & .101 & .098 & 1.00 & .227 & .225 \\
\hline & & .25 & 1.00 & .118 & .119 & 1.00 & .281 & .277 \\
\hline & \multirow{5}{*}{2} & .05 & .993 & .063 & .003 & 1.00 & .235 & .174 \\
\hline & & .10 & 1.00 & .176 & .120 & 1.00 & .481 & .449 \\
\hline & & .15 & 1.00 & .299 & .251 & 1.00 & .721 & .706 \\
\hline & & .20 & 1.00 & .441 & .419 & 1.00 & .852 & .850 \\
\hline & & .25 & 1.00 & .552 & .539 & 1.00 & .935 & .937 \\
\hline & \multirow{5}{*}{3} & .05 & 1.00 & .175 & .019 & 1.00 & .560 & .473 \\
\hline & & .10 & 1.00 & .462 & .375 & 1.00 & .901 & .887 \\
\hline & & .15 & 1.00 & .689 & .651 & 1.00 & .980 & .981 \\
\hline & & .20 & 1.00 & .853 & .840 & 1.00 & .997 & .997 \\
\hline & & .25 & 1.00 & .941 & .935 & 1.00 & .999 & .999 \\
\hline \multirow{20}{*}{$m_{4}$} & \multirow{5}{*}{0} & .05 & .988 & .038 & .001 & 1.00 & .128 & .096 \\
\hline & & .10 & .999 & .042 & .029 & 1.00 & .099 & .096 \\
\hline & & .15 & 1.00 & .040 & .032 & 1.00 & .090 & .083 \\
\hline & & .20 & 1.00 & .047 & .046 & 1.00 & .071 & .071 \\
\hline & & .25 & 1.00 & .036 & .034 & 1.00 & .046 & .049 \\
\hline & \multirow{5}{*}{1} & .05 & .994 & .037 & .001 & 1.00 & .128 & .077 \\
\hline & & .10 & 1.00 & .058 & .038 & 1.00 & .153 & .141 \\
\hline & & .15 & 1.00 & .073 & .058 & 1.00 & .179 & .173 \\
\hline & & .20 & 1.00 & .083 & .082 & 1.00 & .219 & .225 \\
\hline & & .25 & 1.00 & .111 & .104 & 1.00 & .270 & .282 \\
\hline & \multirow{5}{*}{2} & .05 & .998 & .067 & .002 & 1.00 & .240 & .179 \\
\hline & & .10 & 1.00 & .123 & .091 & 1.00 & .488 & .463 \\
\hline & & .15 & 1.00 & .213 & .194 & 1.00 & .715 & .710 \\
\hline & & .20 & 1.00 & .350 & .331 & 1.00 & .849 & .852 \\
\hline & & .25 & 1.00 & .468 & .473 & 1.00 & .929 & .927 \\
\hline & \multirow{5}{*}{3} & .05 & 1.00 & .173 & .019 & 1.00 & .557 & .438 \\
\hline & & .10 & 1.00 & .447 & .352 & 1.00 & .895 & .878 \\
\hline & & .15 & 1.00 & .652 & .616 & 1.00 & .986 & .984 \\
\hline & & .20 & 1.00 & .843 & .842 & 1.00 & .999 & .999 \\
\hline & & .25 & 1.00 & .930 & .932 & 1.00 & 1.00 & 1.00 \\
\hline
\end{tabular}

can see that only minor modification actually improves the performance substantially. For $n=50$, sizes of both tests are little bit smaller than the nominal value of 0.05 and for $n=100$, sizes of both tests approach to 0.05 as $h$ increases. The powers of both test are inevitably low for the small jump $\operatorname{sizes}(d=1)$ but increase rapidly as $d$ increases.

We can notice that the larger the bandwidth is, the more the performance of test improves. In the simulation results of $\mathrm{Wu}$ and $\mathrm{Chu}$ (1993), we can observe the almost same phenomenon. However, this is quite different from the case of the estimation of discontinuity point. According to Qiu (2005), the bandwidth selected for the estimation of the jump position should be chosen relatively small. In the bandwidth selection method 

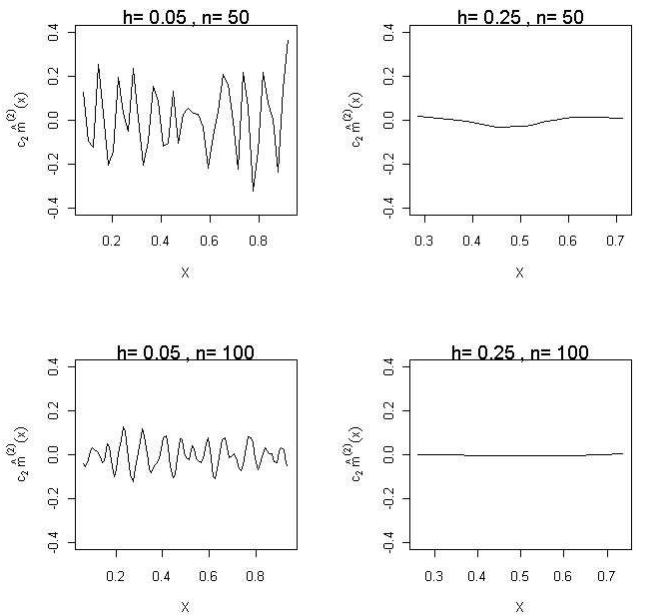

Figure 3.1: $c_{2} \hat{m}^{\prime \prime}(x)$ of the typical data set for $m_{2}$

for the estimation of the jump points of Gijbels and Goderniaux (2004b), they start with $h_{0}$ which is the upper limit of the bandwidth and decrease the bandwidth gradually until a criterion is satisfied. The starting value in their simulation study is always $h_{0}=0.2$.

Note that the rejection ratios of $T_{2}$ and $T_{3}$ are getting closer as $h$ increases. However, for the case of smaller bandwidth, we can notice the significant differences between two tests. Especially, for the case of $h=0.05$ and $n=50$, only a few simulation samples are rejected by $T_{3}$ even in $d=3$.

The reason for this unusual behavior can be explained in Figure 3.1 which present the value of $c_{2} \cdot \hat{m}^{\prime \prime}(x), x \in[h, 1-h]$ for the typical data set of $d=0$ case in the regression function $m_{2}$. Since $m_{2}^{\prime \prime}(x)=0, \hat{m}_{2}^{\prime \prime}(x)$ should be very close to 0 . Moreover, $c_{2}$ itself is a very small value, so the value of $c_{2} \cdot \hat{m}_{2}^{\prime \prime}(x)$ is close to 0 unless $\hat{m}_{2}^{\prime \prime}(x)$ produces enormous value. However, as we can see in Figure 3.1, the value of $c_{2} \cdot \hat{m}_{2}^{\prime \prime}(x)$ for $h=0.05$ is relatively large. This large value of $c_{2} \cdot \hat{m}_{2}^{\prime \prime}(x)$ makes $T_{3}$ small. Thus it is clear that the unusual behavior of $T_{3}$ is induced by instability of $\hat{m}^{\prime \prime}(x)$ at the small value of the bandwidth.

This unstable behavior of $T_{3}$ at small value of $h$ can be a serious problem. Since two one-sided kernel estimators in $T$ can not be defined in the boundary region, $\hat{x}_{0}$ is defined only on the interval $[h, 1-h]$. Therefore, when the regression function has discontinuity points at near each end point of the design space, there is no way to detect them if we use the test statistic with large bandwidth. Thus we prefer the test statistic which can show the stable performance even at the very small bandwidth. In this point of view, we can say that based on our simulation study, $T_{2}$ is better than $T_{3}$.

\section{Conclusion}

A great deal of researches about the jump detector based on nonparametric regression estimator has been done. As we mentioned in Section 2, these statistics can be used as 
the test statistic for the null hypothesis of a smooth regression function. However, only a few statistics were actually proposed as a test statistic. This is because the asymptotic distributions of these test statistics under $H_{0}$ are not accurate at all, so if we rely solely on the asymptotic distribution of the test statistic for determining the critical points, the performance of the test should be very poor.

One way to circumvent this problem can be found in Gijbels and Goderniaux (2004a). They used the bootstrap procedure for determining the critical points of the test. In this paper, we proposed another way to resolve this problem. We argued that the asymptotic distribution of the test statistic could be used for determining the critical points, provided the bias is properly adjusted and proposed the test statistic which include the bias adjustment term. The simulation study shows the possibility that such adjustment really works well.

In fact, the asymptotic distributions of every nonparametric regression estimators are well known and so are the asymptotic distribution of all jump detector based on the nonparametric regression estimator. We need to investigate that the bias adjustment method can work well for all other jump detectors and we will leave this further research topic.

To apply our testing procedure in the real situation, we should have the bandwidth selection rule. Even though we have some data adaptive bandwidth selection method for $\hat{x}_{0}$ and $\hat{m}^{\prime}(x)$, it is not quite clear that these rules work well in the testing problem, too. As we mentioned in Section 3, we may need a relatively large bandwidth, compared with the bandwidth selected for the estimation of the jump positions.

\section{References}

Gijbels, I. and Goderniaux, A. (2004a). Bootstrap test for change-points in nonparametric regression, Journal of Nonparametric Statistics, 16, 591-611.

Gijbels, I. and Goderniaux, A. C. (2004b). Bandwidth selection for changepoint estimation in nonparametric regression, Technometics, 46, 76-86

Müller, H. G. and Stadtmüller, U. (1999). Discontinuous versus smooth regression, The Annals of Statistics, 27, 299-337.

Qiu, P. (2005). Image Processing and Jump Regression Analysis, John Wiley \& Sons, New Jersey.

Wu, J. S. and Chu, C. K. (1993). Kernel type estimators of jump points and values of a regression function, The Annals of Statistics, 21, 1545-1566.

[Received July 2008, Accepted July 2008] 